\title{
MASTALGIA CÍCLICA PRÉ-MENSTRUAL: PLACEBO VERSUS OUTRAS DROGAS
}

\author{
Laurival A. De luca*, Maria de Fátima V. S. Gonçalves, lídia Raquel de Carvalho
}

Trabalho realizado no Centro de Avaliação em Mastologia da Disciplina de Mastologia do Departamento de Ginecologia e Obstetrícia da Faculdade de Medicina de Botucatu, UNESP

*Correspondência

Rua das Plumas, 100

Cep: 18607-270, Botucatu, SP

\section{RESUMO}

Os autores definem mastalgia cíclica pré-menstrual, (MCPM), repassam os principais mecanismos do ciclo celular da mama, e com base nestes conhecimentos propõem a sua classificação em três tipos, segundo a fisiologia do ciclo mamário: tipo I - caracterizado pela distensão localizada de ductos e adensamento do tecido conjuntivo em volta de pequenas dilatações, tipo II - caracterizado pelo edema intersticial, e tipo III - caracterizado pela combinação dos dois processos etiopatogênicos, OBjetivo. Por meio de estudo prospectivo, aleatório, triplo cego e controlado, comparar a ação de placebo com associação de vitaminas A-D-E e doses baixas de ácido acetilsalićlico.

Métodos. Foram observadas 259 portadoras de MCPM, acompanhadas durante seis meses para estudo comparativo das drogas empregadas no alívio da dor. Destas, foram selecionadas 81 pacientes por critérios rigorosos, divididas em três grupos de 27 , que receberam, respectivamente, aspirina, associação de vitaminas e placebo. A dor foi classificada em grau I (sem dor), grau II (dor moderada) e grau III (dor intensa). Os métodos estatísticos realizados mostraram que o número de pacientes em cada grupo era satisfatório. Foi empregado o teste de Tukey para comparação dos resultados e significância a 5\%.

Resultados. As características clínicas, idade, peso, altura e IMC, antecedentes obstétricos e duração da amamentação foram semelhantes nos três grupos. Houve redução de intensidade da dor nos três grupos, principalmente naquele que recebeu placebo.

Conclusä̃o. O estudo realizado, segundo metodologia aceitável, porque foi prospectivo, controlado, triplo cego e aleatório, não mostrou diferenç̧as significativas no tratamento da mastalgia cíclica pré-menstrual entre aspirina e associação de vitaminas, mas revelou superioridade do placebo.

Unitermos: Mastalgia. Mastalgia cíclica. Fisiologia da mama. Tratamento da mastalgia.

\section{INTRODUÇÃO}

MCPM é a dor nas mamas, de caráter cíclico, associada a processos fisiológicos, não relacionada a qualquer causa orgânica, que surge nos dias que antecedem a menstruação e desaparece nos primeiros dias da mesma. Sintoma habitual em consultórios, predomina na adolescência e no menacme. A incidência diminui na pré-menopausa e é nula na menopausa.

Presumíveis alterações hormonais, do sistema reninaangiotensina, de neurotransmissores cerebrais, de enzimas periféricas e carência de determinadas vitaminas, para explicar a etiopatogenia do sintoma, não foram confirmadas em estudos sucessivos. A glândula mamária, na mulher, não permite estudos experimentais; assim, o conhecimento se limita à observação experimental em outros mamíferos, incluindo primatas evoluídos, que não refletem, necessariamente, o que acontece na espécie humana. Trabalhos recentes de histopatolgia, imunogenética e biologia molecular sugerem que, à semelhança do endométrio, passa por modificações cíclicas resultantes do ciclo endócrino ovariano 2,10,16,18,30,31. Resumimos os aspectos básicos das mesmas, para justificarmos a patogenia da MCPM, segundo nossa concepção e que parece justificar certos princípios terapêuticos e rejeitar outros.
Nos primeiros dias da fase estrogênica, inicia-se a proliferação de ductos e de alvéolos. Não há formação de lúmens intraalveolares e o estroma conjuntivo se adensa. A proliferação epitelial se intensifica ao ser instalada a fase lútea, atingindo o pico no auge das produções de estradiol e de progesterona, em torno do $25^{\circ}$ dia $^{32}$. O estradiol exerce ação vaso-dilatadora, parecida com a da histamina sobre a microcirculação do parênquima. A progesterona aumenta a permeabilidade vascular, facilitando a passagem de líquido para o espaço intersticial. Chegam a ser mobilizados $100 \mathrm{~mm}^{3}$ de água para o estroma, aumentando o volume das mamas em cerca de $1530 \mathrm{~mm}^{3(20,25)}$. Dependendo da quantidade do acúmulo de líquido intersticial, pode surgir desconforto acompanhado de sensação de peso ou de distensão das mamas. Classificamos este tipo de mastalgia como MCPM tipo I. Sobrevindo a menstruação, há regressão da proliferação conjuntiva e epitelial, a apoptose é marcante, o fluído intersticial é reabsorvido, o volume volta ao normal e a sensação de desconforto difuso desaparece. Na renovação cíclica, surgem setores de condensação epitelial (com formação de dilatações ducto-alveolares). Resultam pequenas unidades constituídas de dilatações envolvidas por uma túnica de fibroblastos, formando nodularidades milimétricas, que algumas vezes podem ser percebidas pela palpação. Terminações 
nervosas sensitivas comprimidas pelo tecido conjuntivo causam dor em agulhada, em pontada ou latejante, em pontos isolados, ou esparsos em uma ou nas duas mamas, classificamos este tipo de mastalgia como MCPM tipo II. A MCPM tipo III é caracterizada pela associação do desconforto e presença das nodularidades. Durante, ou logo após a menstruação, tais micronódulos se desfazem, os elementos epiteliais e conjuntivos desaparecem e são substituídos por novas células, à medida que se renova o ciclo mamário. Estas modificações estruturais são fisiológicas e explicam por que o exame clínico e a mamografia são prejudicados pela dor e pelo aumento de densidade na semana que antecede a menstruação. $\mathrm{O}$ período ideal para o exame das mamas é a primeira fase do ciclo menstrual, quando só há proliferação celular e ausência dos mecanismos propostos para explicar a dor na segunda fase do ciclo.

A MCPM, enfim, resulta de processo fisiológico (molecular, celular e bioquímico), que constitui a base do tratamento delineado nesta investigação.

\section{Objetivo}

Avaliar a eficácia terapêutica de analgésico tradicional, em baixas doses, comparada ao uso de associação de vitaminas A, B6 e D, com placebo, na atenuação ou desaparecimento da MCPM, tendo como premissa que o sintoma é resultado de processo fisiológico que ocorre no ciclo da mama.

\section{Métodos}

Entre março de 2000 e março de 2003, foram observadas 259 portadoras de MCPM, acompanhadas durante dois meses prévios ao tratamento, e durante seis meses, para estudo comparado do tratamento. Foram excluídas 178 porque não se ajustaram aos critérios de inclusão. Oitenta e uma mulheres foram acompanhadas porque obedeceram os seguintes critérios de inclusão:

- Idade maior que 18 anos;

- Ciclos menstruais normais com intervalos regulares entre 21 e 35 dias;

- Mastalgia pré-menstrual por mais de sete dias por mês, em pelo menos dois meses consecutivos;

- Data da última menstruação inferior a 30 dias;

- Nenhuma medicação usada nos últimos dois meses, inclusive contraceptivos hormonais orais ou injetáveis;

- Não referir antecedentes de alergia a vitaminas ou ácido acetil salicílico (AAS);

- Ter recebido consentimento informado sobre a metodologia e objetivos da investigação.

Critérios de exclusão

- Abandono do tratamento nos dois primeiros meses;

- Recusa em aceitar o tratamento sem conhecimento prévio;

- Diagnóstico de gravidez;

- Uso inadequado da medicação;

- Diagnóstico de lesão suspeita em uma das mamas;

- Nódulos maiores do que 10 mm;

- Uso prévio de contraceptivos hormonais orais.

Todas foram submetidas a rigorosos anamnese e exame clínico e exame ultra-sonográfico de alta resolução das mamas (Siemens, VersaPró, com transdutor linear de $10 \mathrm{MHz}$ ).

O estudo foi prospectivo, aleatório, triplo cego e placebo controlado. As medicações AAS, associação de vitaminas A, B6 e D, e o placebo foram acondicionadas em recipientes idênticos, rotulados com as letras A, B e C, cujo conteúdo era desconhecido.

A distribuição dos recipientes, cujo conteúdo não era do conhecimento nem do examinador nem da paciente, foi feita após as consultas preliminares, de forma aleatória. As pacientes que completaram o período integral de tratamento (três meses) após visitas mensais, foram divididas em três grupos, depois da identificação das medicações contidas nos respectivos recipientes:

Grupo A: 27 pacientes que tomaram AAS;

Grupo B: 27 pacientes que tomaram placebo;

Grupo C: 27 pacientes que tomaram a associação de vitaminas.

A dor foi avaliada mediante graduação simples:

Grau I = nenhuma dor;

Grau II = dor moderada;

Grau III = dor intensa

As medicações foram prescritas segundo o esquema:

Grupo A: AAS = comprimidos de $250 \mathrm{mg}$;

Grupo B: lactose = comprimidos de $20 \mathrm{mg}$ de lactose;

Grupo C: associaçãa de retinol (5.000 UI), cloridrato de piridoxina ( $100 \mathrm{mg}$ ) e acetato de tocoferol (300 mg)

As pacientes dos três grupos receberam as medicações e o placebo de modo idêntico: dois comprimidos ao dia, durante 10 dias antes da data provável da menstruação.

Para a análise estatística, o tamanho amostral mínimo foi determinado em 27 pacientes para cada grupo. Utilizou-se o nível de 5\% de significância. O poder de teste foi de $80 \%$ e uma diferença esperada entre o percentual de melhora dos grupos tratados em relação ao placebo em torno de 30\%. Foi utilizado o teste de Tukey para comparação dos percentuais referentes à graduação da dor. O nível de significância utilizado foi de $5 \%{ }^{34}$.

\section{Resultados}

A média de idade das pacientes foi de 4 I anos. Peso, altura, IMC, idade da menarca, idade do primeiro parto, número de gestações de abortos e de partos e a duração da amamentação não apresentavam diferenças significativas nos três grupos (valor de p entre 0,05 e 0,99).

Graduação da intensidade da dor antes e depois do tratamento: Grupo I (AAS)

Antes do tratamento:

Grau II = $08(29,6 \%)$

Grau III = $19(70 \%)$

Após o tratamento:

Grau I = $13(48,1 \%)$

Grau $\|=10(32 \%)$

Grau III = $4(14,9 \%)$

Grupo II (placebo)

Antes do tratamento:

Grau $\|=07(25,9 \%)$

Grau III $=20(74,1 \%)$ 


\begin{tabular}{|c|c|c|c|c|}
\hline \multicolumn{5}{|c|}{$\begin{array}{l}\text { Tabela I - Média e desvio padrão referentes às variáveis de } \\
\text { caracterização das pacientes segundo do grupo }\end{array}$} \\
\hline & \multicolumn{4}{|c|}{ Grupos } \\
\hline & A & B & C & Valor de $\mathrm{p}$ \\
\hline Peso & $61,8 \pm 12,4$ & $62,2 \pm 10,6$ & $69,4 \pm 14,5$ & 0,05 \\
\hline Altura & $1,57 \pm 0,06$ & $1,56 \pm 0,06$ & $1,59 \pm 0,05$ & 0,08 \\
\hline Idade & $42,7 \pm 9,0$ & $40,3 \pm 13,4$ & $4|,| \pm \mid 1,5$ & 0,75 \\
\hline IMC & $25,2 \pm 5,3$ & $25,3 \pm 3,8$ & $27,0 \pm 5,0$ & 0,31 \\
\hline Idade menarca & $12,9 \pm 2,2$ & $13,2 \pm 2,0$ & $12,8 \pm 1,8$ & 0,75 \\
\hline Idade $1^{\circ}$ parto & $21,1 \pm 3,4$ & $21,0 \pm 5,7$ & $21,9 \pm 4,4$ & 0,75 \\
\hline $\mathrm{N}^{0}$ de gestações & $3,4 \pm 2,4$ & $2,8 \pm 1,8$ & $2,5 \pm 1,2$ & 0,27 \\
\hline $\mathrm{N}^{0}$ de partos & $2,8 \pm 1,7$ & $2,8 \pm 1,8$ & $2,3 \pm 0,9$ & 0,37 \\
\hline $\mathrm{N}^{0}$ de abortos & $0,5 \pm 1,0$ & $0,5 \pm 0,9$ & $0,5 \pm 0,8$ & 0,99 \\
\hline Duração da lactação & $1,4 \pm 1,4$ & $1,8 \pm 2,3$ & $1,8 \pm 2,3$ & 0,74 \\
\hline
\end{tabular}

Após o tratamento:

Grau I = 23 (85\%)

Grau $\|=0$

Grau III = $04(\mid 4,8 \%)$

Grupo III (associaçãa de vitaminas)

Antes do tratamento:

Grau $\|=10(37 \%)$

Grau III = $17(63 \%)$

Após o tratamento:

Grau I = |4 (51,8\%)

Grau II $=09(33,3 \%)$

Grau III = $04(14,8 \%)$

Número de pacientes com remissão ou atenuação da mastalgia, nos três grupos:

Grupo I (AAS) $=23(85,1 \%)$

Grupo II (placebo) $=23(85,1 \%)$

Grupo III (associação de vitaminas) = 23 (85, I\%)

Número de pacientes com remissão completa da mastalgia:

Grupo I (AAS) $=13(48,1 \%)$

Grupo II (placebo) $=23(85 \%)$

Grupo III (Associação de vitaminas) = 14 (51,8\%)

Número de pacientes com atenuação da mastalgia:

Grupo I (AAS) $=10(32 \%)$

Grupo II (placebo) $=0 \%$

Grupo III (associação de vitaminas) $=9$ (33,3\%)

Estes resultados mostraram diferenças significativas da graduação da dor, nos dois grupos, antes e depois do tratamento $(p<0,05)$.

Além das 27 pacientes de cada grupo que completaram o estudo, 5 do grupo I, 7 do grupo II e I 2 do grupo III abandonaram o tratamento. As pacientes dos três grupos foram observadas durante outros três meses, sem medicação. Em todos eles, observamos que os sintomas mostravam-se atenuados, mesmo após a interrupção do tratamento. As Tabelas I a 5 refletem os resultados abordados.

\begin{tabular}{|c|c|c|c|}
\hline \multicolumn{4}{|c|}{ Tabela 2 - Grupo AAS } \\
\hline Pré-tratamento & Score I & Score 2 & Score 3 \\
\hline Ia visita & $\overline{70,40 \%(19)}$ & $\overline{29,60 \%(08)}$ & \\
\hline $2^{\mathrm{a}}$ visita & $63,00 \%(17)$ & $37,00 \%(10)$ & \\
\hline \multicolumn{4}{|l|}{ Com medicação } \\
\hline$I^{\mathrm{a}}$ consulta & $26,00 \%(07)$ & $55,50 \%(\mid 5)$ & $18,50 \%(05)$ \\
\hline $2^{\mathrm{a}}$ consulta & $14,90 \%(04)$ & $44,40 \%(12)$ & $40,70 \%(10)$ \\
\hline $3^{\mathrm{a}}$ consulta & $14,90 \%(04)$ & $37,00 \%(10)$ & $48,10 \%(13)$ \\
\hline \multicolumn{4}{|c|}{ Tabela 3 - Grupo placebo } \\
\hline Pré-tratamento & Score I & Score 2 & Score 3 \\
\hline I visita & $\overline{74,10 \%(20)}$ & $\overline{25,90 \%(07)}$ & \\
\hline $2^{a}$ visita & $74,10 \%(20)$ & $25,90 \%(07)$ & \\
\hline \multicolumn{4}{|l|}{ Commedicação } \\
\hline$I^{a}$ consulta & $\mid 4,80 \%(04)$ & $62,90 \%(17)$ & $22,30 \%(06)$ \\
\hline $2^{\mathrm{a}}$ consulta & $7,50 \%(02)$ & $44,40 \%(12)$ & $48,10 \%(13)$ \\
\hline $3^{\mathrm{a}}$ consulta & $14,80 \%(04)$ & $00,00 \%(00)$ & $85,19 \%(23)$ \\
\hline
\end{tabular}

Tabela 4 - Grupo palmitato de retinol 5000 ui, cloridrato de piridoxina $100 \mathrm{mg}$, acetato de tocoferol $300 \mathrm{mg}$

\begin{tabular}{lccc}
\hline $\begin{array}{l}\text { Pré-tratamento } \\
I^{\mathrm{a}} \text { visita }\end{array}$ & \multicolumn{1}{c}{ Score I } & \multicolumn{1}{c}{ Score 2 } & Score 3 \\
$2^{\mathrm{a} v \text { visita }}$ & $63,00 \%(17)$ & $37,00 \%(10)$ & \\
Com medicação & $44,40 \%(12)$ & $55,60 \%(15)$ & \\
$I^{\mathrm{a} \text { consulta }}$ & $33,40 \%(09)$ & $55,56 \%(15)$ & $11,10 \%(03)$ \\
$2^{\mathrm{a}}$ consulta & $26,00 \%(07)$ & $29,60 \%(08)$ & $44,40 \%(12)$ \\
$\mathrm{J}^{\mathrm{a}}$ consulta & $14,90 \%(04)$ & $25,90 \%(07)$ & $59,20 \%(12)$ \\
\hline
\end{tabular}

\begin{tabular}{lcc}
\hline \multicolumn{3}{c}{ Tabela 5 - Adesão ao tratamento } \\
\hline Fórmula & $\begin{array}{c}\text { Pacientes avaliadas } \\
\text { até o final do estudo }\end{array}$ & $\begin{array}{c}\text { Pacientes que } \\
\text { abandonaram } \\
\text { o tratamento }\end{array}$ \\
\cline { 2 - 3 } & 27 & 5 \\
AAS $250 \mathrm{mg}$ & 27 & 7 \\
Placebo & 27 & 12 \\
Acetato de retinol $5000 \mathrm{UI}$ & & \\
Vitamina E & & \\
Piroxidina $100 \mathrm{mg}$ & & \\
\hline
\end{tabular}

\section{Discussão}

A hipótese proposta que classifica a MCPM ${ }^{7}$ em tipos I e II, embora não possa ser comprovada em anima nobile, encontra respaldo em conhecimentos fisiológicos que regem as modificações das estruturas mamárias durante o ciclo menstrual. A escolha do tratamento com analgésicos simples e com placebo decorre desta hipótese. A investigação de três grupos homogêneos de pacientes realizada de forma aleatória, prospectiva, triplo cega e controlada, com rígidos critérios de inclusão, sugerem que o tratamento é meramente sintomático, dispen- 
sando medicamentos de alto custo com efeitos colaterais e que não respeitam a fisiologia do ciclo mamário. A média de idade nos três grupos (4I anos) é compatível com a incidência encontrada na literatura, situando-a em torno de 40 anos.

A base do tratamento consiste no suporte emocional e orientação verbal, mostrando que a dor se origina em processos normais e que nada tem a ver com câncer. Conforme já assinalamos, o tratamento medicamentoso habitual é múltiplo e diversificado, mas nenhum encontra respaldo na fisiologia. Alguns são muito caros, outros têm respostas efêmeras ou causam efeitos colaterais indesejáveis. O uso de diuréticos é discutível, pois a retenção hídrica intersticial, possivelmente, não guarda relação com a água total do organismo e não passa pela excreção renal. O ácido gama-linolêico, precursor da prostaglandina $E$, apesar de não causar efeitos colaterais, é de alto custo e tem ação inibidora sobre a prolactina que não está envolvida no mecanismo da dor; seus efeitos terapêuticos não ultrapassam $44 \%{ }^{27}$. O danazol e a gestrinona têm preço proibitivo, causam efeitos colaterais importantes, não consultam o mecanismo da dor, sendo a nosso ver contraindicados. As mesmas considerações valem para a bromoergocriptina. Os vários tipos de progestagênios, medicação de maior mercado e utilização, até há poucos anos, são inúteis, contra-indicados, anti-fisiológicos e não demonstram efeito superior ao placebo'. Análogos dos fatores de liberação das gonadotrofinas para tratamento da MCPM, deveriam ser proibidos. Ainda que atenuem a dor em $81 \%{ }^{15}$ dos casos, agem por meio de inaceitável inibição da função ovariana. 0 tamoxifeno, em doses de 10 ou $20 \mathrm{mg}$, pode resultar em atenuação ou remissão completa da dor em muitos casos ${ }^{9}$. Sua ação antiestrogênica responde, parcialmente, pelo seu mecanismo de ação, que é a inibição da proliferação e possível redução da vaso-dilatação na microcirculação. O problema com o tamoxifeno são os efeitos colaterais, o preço, e a contra-indicação do uso por mais de três a seis meses. Ao contrário dos autores que defendem seu uso, não cremos que a droga, após ser interrompida, impeça a recidiva dos sintomas, porque esperase a reversão fisiológica dos mecanismos que os causam. Inibidores da recaptação de serotonina são indicados quando a mastalgia se associa à ansiedade e outras manifestações de depressão que fazem parte da síndrome pré-menstrual22.

O ácido acetilsalicílico tem ação analgésica, periférica e central. Inibe a síntese de prostaglandinas, impede a sensibilização dos algoreceptores ao estímulo mecânico ou químico. Suas contra-indicações (alergia, irritação gástrica, bronespasmo, urticária, edema angio-neurótico) são raras, mas devem ser questionadas na anamnese. A dose analgésica varia entre 100 e $500 \mathrm{mg}$ a cada quatro horas, não se devendo ultrapassar 4 a $8 \mathrm{~g} /$ dia. Nesta investigação, usamos doses muito baixas de $500 \mathrm{mg}$, divididas em $250 \mathrm{mg} / \mathrm{dia}$. A associação de vitaminas A, B6 e D tem sido recomendada com a suposição de estabelecer a integridade das células conjuntivas e epiteliais, promovendo a diferenciação e amadurecimento celular ${ }^{19,33}$. Esta hipótese não tem qualquer relevância na etiopatogenia da MCPM. Usados durante dez dias, nas doses indicadas, nem a aspirina nem as vitaminas apresentaram efeitos colaterais e resultaram em resultados terapêuticos satisfatórios e semelhantes. No grupo II, as pacientes foram tratadas com placebo, lactose quimicamente inerte, sendo a resposta terapêutica significativamente melhor do que nos outros dois grupos. É relevante o fato de as pacientes terem tido apoio psicológico e atenção, que podem ter beneficiado os resultados, inclusive quando encerrado o período de comparação dos medicamentos com a suspensão destes. Também é possível que doses maiores de aspirina pudessem ter efeito mais potente no alívio da mastalgia. Esta investigação parece mostrar que a MCPM é passível de tratamento sintomático e barato, enfatizando o efeito placebo. Não há necessidade de medicamentos de alto custo, com efeitos colaterais e que não consultam o mecanismo fisiológico da dor.

\section{Conclusão}

O estudo comparativo, triplo cego, aleatório, prospectivo e controlado de ácidoacetilsalićlico, associação de vitaminas (A, B6, D) e placebo não mostrou diferenças significativas no tratamento da MCPM, entre os dois primeiros medicamentos, mas revelou superioridade significativa do placebo.

\section{Agradecimentos}

Agradecemos à colaboração da Srta. Kátia Regina de Caria na apresentação datilografada e em várias sugestões complementares.

\section{Conflito de interesse: não há}

\section{SUMMARY}

\section{Pre-menstrual cyclic mastalgia}

OBIECTNES. To compare therapeutic effects of placebo with intake oflow doses of acetyl-salysilic acid (aspirin) and a compound of retinol, pyridoxine chlorhydrate and tocopherolacetate.

METHODS. The study is a prospective, controlled, triple blinded, randomized trial. From 259 patients, 81 were selected in order to follow rigid criteria of inclusion. They were divided into three groups of 27 patients, taking aspirin, vitamins or placebo respectively. The number of patients in each group was considered satisfactory for statistical analysis. Pain was scored as Grade I (without pain), Grade II (moderate pain) and Grade III(severe pain). The Tukeytestwas appliedforcomparison of results (statistical significance 5\%).

RESULTS. Clinical parameters, age, weight, BMI, parity and lactation period were similar for each group. There was a decrease of pain intensity in all groups, that was even more pronounced for the placebogroup.

DISCUSSION. Emphasis was given to symptomatic treatment of cyclic premenstrual mastalgia with symptoms considered to be the expression of a physiological process that can be treated with simple drugs or placebo. Usual therapeutic approaches with several drugs are criticized, including results and contraindications.

CONCLUSION. This study based upon acceptable trial methodology (randomized, triple blinded, placebo controlled, prospective) did not show significant differences in the treatment of cyclic mastalgia. [Rev Assoc Med Bras 2006; 52(4): 265-9]

KEY WORDS: Mastalgia. Mammary cycle. Cyclic mastalgia 


\section{REFERÊNCIAS}

I. Azzopardi JG. Problems in breast pathology. Philadelphia: W. B. Saunders Co., 1979

2. Coltrera MD, Wan J. Expression of PDGF B-chain and the platelets derived growth factor receptor Beta subunit in human breast tissue and breast carcinoma. Cancer Res 1995;55:2702-8.

3. De Luca LA. Considerações sobre doenças benignas da mama. Bol Reg São Paulo (SBM) 2003; Junho.

4. Fentiman IS. Management of breast pain. Harris JR, Lippman ME, Morrow M, editors. Diseases of the breast. $3^{\text {rd }}$ ed. Lippincot: Williams and Williams; 2004

5. Gately CA, Mansel RE. Management of cyclic breast pain. Br J Hosp Med 1990; 43:330-2.

6. Fergunson DJP, Anderson TJ. An ultrastructural study of mitosis and cytokinetics in normal resting human breast. Cell Tissue Res 1988; 252:58I-7.

7. Hamed H, Callefi M. LHRH analogue for treatment of recurrent and refratory breast pain. Ann R Coll Surg Engl 1990;72: 221-4.

8. Hayes DF. Atlas of breast cancer. St Louis: Mosby Co; 2000.

10. Khanna AK, Tapodar J, Misra MK. Spectrum of benign breast diseases in a University Hospital. J Indian Med Assoc 1997;95:5-8.

I I. Louro ID, Llerena J, Melo S. Genética molecular do câncer MSG. São Paulo: Produção Editorial; 2002.

12. Maddox PR, Harrison BJ, Horobin JM. A randomized controlled trial of medroxiprogesterone acetate in mastalgia. Ann R Coll Surg Engl 1990;72: 7|-6.
13. Milligan D, Drife J, Short RV. Changes in breast volume during normal menstrual cycle and after oral contraceptives. Br Med J 1975;4:494-6.

14. Peters F, Diemer $P$, Meckes $O$. Severity of mastalgia in relation to milk dilatation. Am J Obstet Gynecol 2003; 1 0 1:54-72.

15. Rapkin AJ. The role of serotonin in premenstrual syndrome. Clin Obstet Gynecol 1992;35:629-36.

16. Robbins SL, Cotran RS. Pathologic basis of disease. $7^{\text {th }}$ ed. Amsterdan: Elservier-Saunders; 2005.

17. Rosen PP. Breast pathology. Philadelphia: Lippincott, Williams and Williams; 2001.

18. Secreto C. Fisiopatologia della mammella. Milano: Ed. Masson; 1999. Tavassoli FA. Pathology of the breast. New York: Appleton. LangeStanford; 2002.

19. Vogel OM. The correlation of histologic changes in the human breast with menstrual cycle. Am J Pathol 1981; 104: 23-5.

20. Wetzig NR. Mastalgia: a three year Australian study. Aust N Z J Surg |994:64:329-31.

21. Zar JH. Biostatistical analysis. Upper Saddle River: Prentice Hall; 1999.

Artigo recebido: 08/04/05

Aceito para publicação: I //02/06 